\title{
A 5.2nV/VHz Noise Density Third-Order FDNR Filter For DVB-H Mobile-TV Receiver
}

Conference Paper · December 2013

CITATION

1

3 authors, including:
READS

33

Soliman A. Mahmoud

University of Sharjah, UAE/Fayoum University, Egypt

184 PUBLICATIONS 1,239 CITATIONS

SEE PROFILE

Some of the authors of this publication are also working on these related projects:

Project

Field Programmable Analog Array View project

Project

EEG Detection System View project

All content following this page was uploaded by Soliman A. Mahmoud on 13 September 2015.

The user has requested enhancement of the downloaded file. All in-text references underlined in blue are added to the original document and are linked to publications on ResearchGate, letting you access and read them immediately. 


\title{
A $5.2 \mathrm{nV} / \sqrt{ } \mathrm{Hz}$ Noise Density Third-Order FDNR Filter for DVB-H Mobile-TV Receiver
}

\author{
Soliman Mahmoud, Aisha Abdallah and Sara Khalid \\ Department of Electrical and Electronics Engineering UOS \\ Sharjah, U.A.E. \\ Email: \{solimanm, U00010746, U00013269\}@sharjah.ac.ae
}

\begin{abstract}
In this paper, noise-shaping gain-filtering techniques which minimize noise over the range of passband filter have been studied. These techniques will be demonstrated by applying it on a part of CMOS mobile-TV receiver. This paper mainly focuses on designing a low pass filter, consisting of two cascaded parts. The first part is Frequency Dependent Negative Resistance (FDNR) based third-order noise shaped pre-filter section, which has DC gain of $80 \mathrm{~dB}$, with cutoff frequency of $818 \mathrm{KHz}$ and input referred noise voltage power spectral density of $5.2 \mathrm{nV} / \mathrm{sqrt}(\mathrm{Hz})$. The second part is Asymmetric Floating Frequency Dependent Negative Resistance (AFFDNR) based feedback network, which is a part of instrumentation amplifier. It has DC gain of $20 \mathrm{~dB}$, with cutoff frequency of $765 \mathrm{KHz}$ and input referred noise voltage power spectral density of 83nV/sqrt(Hz). These two filters depend on CMOS op amp which has DC gain of $82 \mathrm{~dB}$, with unity gain bandwidth of $319 \mathrm{MHz}$, phase margin of $61^{\circ}$ and power dissipation of $428 \mu \mathrm{W}$. This CMOS op amp is designed based on $0.25 \mu \mathrm{m}$ CMOS technology with power supply of $\pm 1.5 \mathrm{~V}$.
\end{abstract}

\section{INTRODUCTION}

One of wireless applications which occupy an important position in communication is mobile-T.V. In such system, the received signal is usually affected by very strong interferes that emerge from adjacent channels [1]-[2]. Based on this concept, a wireless receiver's system demands high requirements to achieve the original signal. One of these requirements is filtering at the receiver. Noise-shaping gainfiltering techniques are one of the preferable filtering methods used at these receivers. This work emulates reference [1] but the challenge is in the technology. In reference [1], the design is done in 65-nm CMOS. So, this leads to any difference between desired and simulated values.

In Section II, the standard response of elliptical filter is described. Section III discusses specifications of proposed CMOS op amp along with simulation results. Section IV presents FDNR filter and AFFDNR gain filtering stages with the designed results. Section V summarizes the conclusion.

\section{ELLIPTICAL FILTER STANDARD RESPONSES}

The elliptic filter is the only filter that has equi-ripple behavior in both passband and stopband regions. It has the sharpest transition region among all filters. As the order of the elliptic filter increases, its transition region becomes smaller.

\section{A. The Transfer Function}

The elliptic filter is a pole-zero filter and both the numerator and denominator of the transfer function are polynomials. Coefficients of the polynomials can be found either by using the coefficient table or MATLAB commands. Coefficients can be found using [3]

$$
\begin{gathered}
H_{N}(s)=\frac{H_{0}}{D_{N}(s)} \prod_{i=1}^{r} \frac{s^{2}+A_{0 i}}{s^{2}+B_{1 i} s+B_{0 i}} \\
D_{N}(s)=\left\{\begin{array}{ll}
s+s_{o}, & \mathrm{~N} \text { odd } \\
1, & \mathrm{~N} \text { even }
\end{array} \mathrm{r}=\left\lfloor\frac{N}{2}\right\rfloor .\right.
\end{gathered}
$$

Designing a third order elliptic filter for passband gain equals $1 \mathrm{~dB}$ and stopband gain equals $-30 \mathrm{~dB}$ can be written as

$$
H_{3}(s)=\frac{H_{0}\left(s^{2}+A_{0}\right)}{s^{3}+\left(s_{0}+B_{1 i}\right) s^{2}+\left(s_{0} B_{1 i}+B_{0 i}\right) s+B_{0 i}} .
$$

\section{B. Designing Elliptic Filters}

There are two approaches to design third order elliptic filter according to given parameters either by having a certain order or by having a set of specifications. These approaches start from having normalized elliptic filter besides having specific parameters and end by obtaining denormalized elliptic filter. The normalized form of an odd order elliptic filter is [3]

$$
H_{n}(s)=\frac{H_{0}}{\left(s+s_{0}\right)} \prod_{i=1}^{(n-1) / 2} \frac{s^{2}+A_{0 i}}{s^{2}+B_{1 i} s+B_{0 i}}, \text { odd n. }
$$

There are two approaches in designing elliptic filters. In the first approach, the order of the filter is known. The given transfer function must be written as (1). The following parameters $\omega 1$ and $\omega r$ can be found from the table by knowing the order. The normalized frequency is calculated to find the unnormalized cutoff frequency of the stopband [3]. Finally, each (s) is replaced by ( $\mathrm{s} / \omega \mathrm{o})$ to have an equation as below

$$
\frac{H_{0}\left(s / \omega_{0}\right)^{2}+\left(H_{0} \cdot A_{0}\right)}{\left(s / \omega_{0}\right)^{3}+\left(B_{1 i}+s_{0}\right)\left(s / \omega_{0}\right)^{2}+\left(B_{0 i}+B_{1 i} \cdot s_{0}\right)\left(s / \omega_{0}\right)+\left(B_{0 i} \cdot s_{0}\right)} .
$$

This process is called transformation from low pass to low pass. By using the table of elliptic filter, the coefficients can be found, thus normalized transfer function of the third order is obtained. 


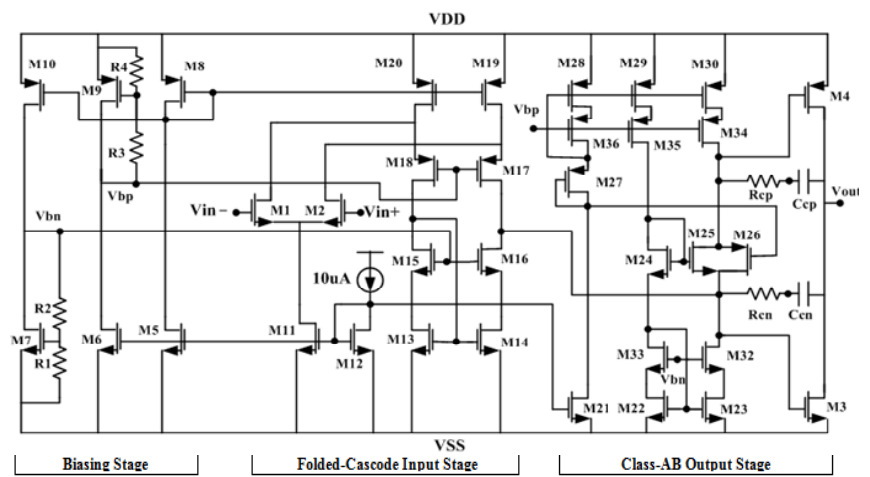

Fig. 1. The schematic of CMOS op amp [1]

The second approach is based on designing a normalized filter to match G1, G2 and $\omega r$. By having these parameters, the proper table is selected and the order of the filter is figured out. After using (1) and substituting by coefficients, the normalized transfer function is established. The values of $\omega 1$ and $\omega 2$ will depend on the value of $\omega r$ [3].

\section{THE PROPOSED CMOS OP AMP}

The CMOS op amp shown in Fig. 1 is used in the designed filters. It consists of three stages; biasing stage, folded-cascode input stage and class- $\mathrm{AB}$ output stage. The biasing stage is designed based on threshold voltage multiplier which allows using simple voltage divider equations [4]. It biased the PMOS and NMOS in CMOS op amp. The input stage has a structure of a folded-cascode, which folds down P-channel cascode active loads of a differential pair and changing the MOSFETs to N-channels [5]. The class-AB output stage has three main blocks; the wide-swing current mirror, floatingcurrent source and class- $\mathrm{AB}$ structure. The wide-swing is used to bias the floating-current source, while floating-current source is used to bias class- $\mathrm{AB}$. The class- $\mathrm{AB}$ main feature is low power consumption and good linearity [6]. Table I presents specifications of designing CMOS op amp. The simulation is done using PSPICE A/D Candence version 16. Table II summarizes simulated results, and the figures of each parameter are shown in Fig. 2-3.

\section{THIRD ORder NoISE SHAPED Elliptic Filter AND AFFDNR GAIN-FILTERING STAGES}

An FDNR is an active element which was designed by Bruton in 1970 [1]. It derives from GIC (Generalized Impedance Converter) structure, which was built by Antonion in 1970 [7]. A grounded FDNR based third order elliptic filter is used in mobile-T.V. receiver. By using a floating FDNR structure, an AFFDNR based on feedback network is established as a stage after FDNR based filter. It works as an amplifier-filtering stage with the corporation of PMA [1].

\section{A. The Realization of Grounded FDNR}

A grounded FDNR can be realized from GIC design. The GIC circuit consists of four impedances with two op amps. It represents as two-port network which its transmission matrix can be formed as (6), and its input impedance is given in (7). If $\mathrm{B}$ and $\mathrm{C}$ parameters are zero, this leads to have converter network [7] which is given by (8).
TABLE I. CMOS OP AMP SPECIFICATIONS

\begin{tabular}{|c|c|}
\hline Parameters & Expected values \\
\hline DC-gain & $>60 \mathrm{~dB}$ \\
\hline Unity Gain Bandwidth & $>200 \mathrm{MHz}$ \\
\hline Phase margin & $61^{\circ}$ \\
\hline Power dissipation & $<500 \mu \mathrm{W}$ \\
\hline
\end{tabular}

TABLE II. CMOS OP AMP RESULTS AFTER SIMULATION

\begin{tabular}{|c|c|}
\hline Parameters & Simulation values \\
\hline DC-gain & $81.819 \mathrm{~dB}$ \\
\hline Unity Gain Bandwidth & $319.202 \mathrm{MHz}$ \\
\hline Phase margin & $61.167^{\circ}$ \\
\hline Power dissipation & $428.466 \mu \mathrm{W}$ \\
\hline
\end{tabular}

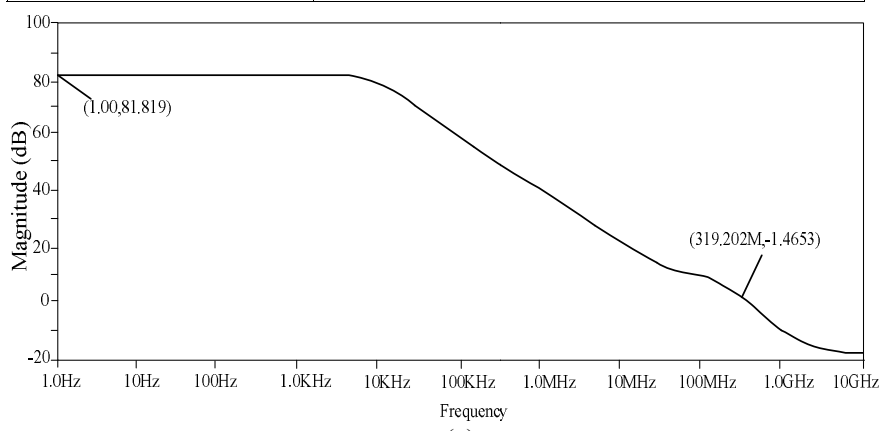

(a)

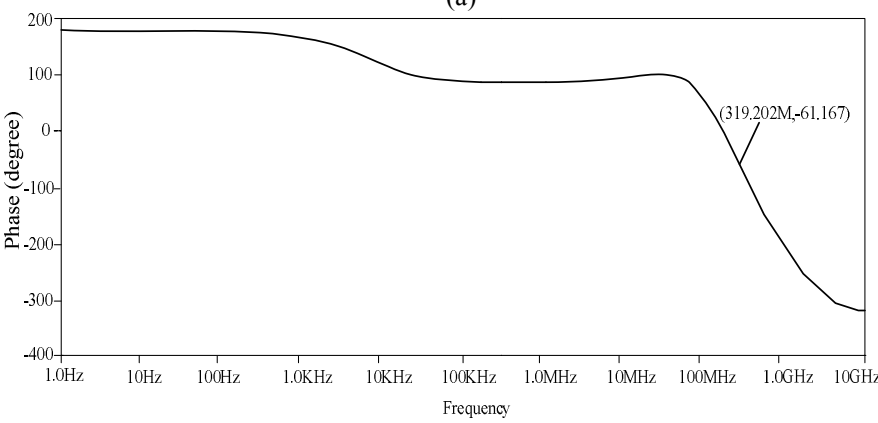

(b)

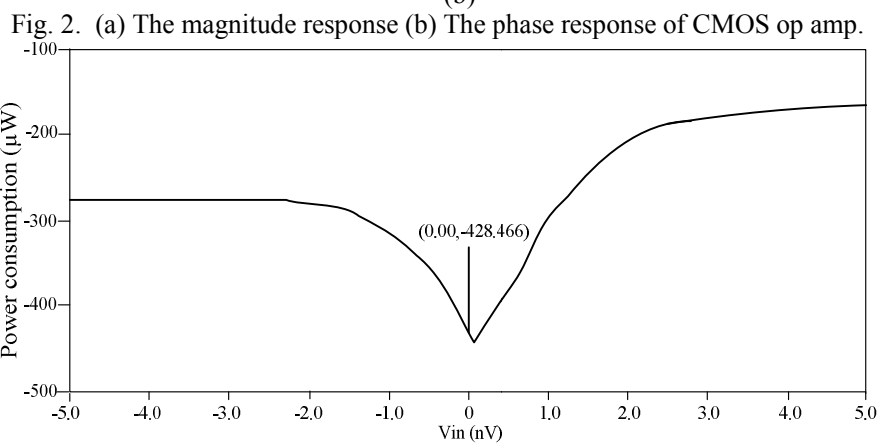

Fig. 3. The power consumption of CMOS op amp.

$$
\begin{aligned}
{\left[\begin{array}{l}
V_{1} \\
I_{1}
\end{array}\right] } & =\left[\begin{array}{ll}
A & B \\
C & D
\end{array}\right]\left[\begin{array}{c}
V_{2} \\
-I_{2}
\end{array}\right] \\
Z_{\text {in }} & =\frac{A Z_{L}+B}{C Z_{L}+D} \\
Z_{\text {in }} & =\frac{A Z_{L}}{D} .
\end{aligned}
$$


An FDNR element can be founded using transmission matrix working as converter network where input impedance is obtained in (9).

$$
Z_{\text {in }}=\frac{Z_{1} Z_{3} Z_{5}}{Z_{2} Z_{4}}
$$

Equation (9) indicates three choices to create a grounded FDNR. It can be designed by two of impedances in numerator are selected to be capacitors and rest of impedances is resistors. This design produces an element called grounded D that has same symbol of capacitor but with three plates shown in Fig. 4. Grounded D element and input impedance can be written as [7]

$$
\begin{gathered}
D=C^{2} r \\
Z_{\text {in }}=1 / s^{2} D .
\end{gathered}
$$

\section{B. The FDNR Based Filter}

The noise shaping technique target is to design filter with lower noise using same component values of traditional filters. In this concept, usage of FDNR as based noise filter section comes as one of the modern inventions. FDNR based third order low pass filter in Fig. 5 provides noise shaping features which used to design low noise. The main advantage of using a grounded FDNR is offering only one resistor in the signal path to realize high order filters [1], where noise shaping is done by $\mathrm{C} 1$. At low frequencies, capacitors, $\mathrm{C} 1$ and $\mathrm{C} 2$, are open circuit and the only path is through resistor Rf. At high frequencies, $\mathrm{C} 1$ and $\mathrm{C} 2$ offer a path for the signal with low impedance. The transfer function of the third-order elliptic circuit is given by [1]

$$
\frac{V_{\text {out }}(s)}{I_{\text {in }}(s)}=\frac{R_{f}\left(s^{2} D R_{\mathrm{Z}}+1\right)}{s^{3} D R_{\mathrm{Z}} R_{f} C_{f}+s^{2}\left(D R_{\mathrm{Z}}+D R_{f}\right)+s\left(R_{f} C_{f}\right)+1}=\frac{R_{f} \cdot N(s)}{D(s)}
$$

Where

$$
D=\frac{C_{1} C_{2} R_{1} R_{3}}{R_{2}} .
$$

$\mathrm{N}(\mathrm{s})$ is transfer function with notch frequency $\omega_{\text {notch }}=\frac{1}{\sqrt{D R_{Z}}}$.

$\mathrm{N}(\mathrm{s})$ and $\mathrm{D}(\mathrm{s})$ are closed to unity in the passband region and the transfer function can be represented only by $R_{f}$.

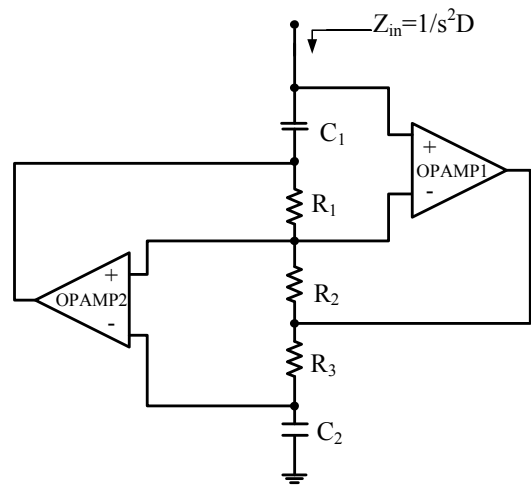

(a)

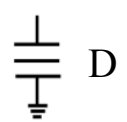

(b)
Fig. 4. (a) The equivalent circuit of grounded FDNR, (b) its symbol.

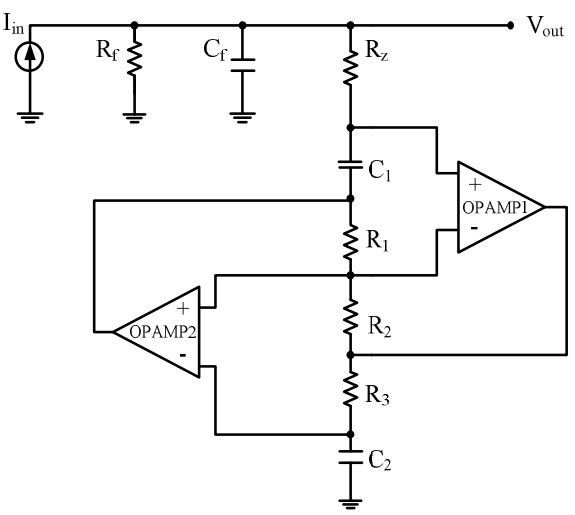

Fig. 5. FDNR based third order low pass filter.

\section{AFFDNR Based Feedback Network}

An FDNR can be realized as a floating element by using a GIC. By finding equivalent impedances at the two ports of GIC, it can be shown that two impedances are not the same. At node $\mathrm{A}$, the impedance $\mathrm{ZA}$ is negative when node $\mathrm{B}$ grounded, while the impedance at node $\mathrm{B}, \mathrm{ZB}$, is capacitive when node $A$ is grounded, so it is called Asymmetric Floating FDNR [2]. The AFFDNR works as gain-filtering stage with PMA for instrumentation topology as show in Fig. 6.

\section{The Simulation Results}

Designing pre-filter FDNR and amplifier cascade filter depend on the design of op amp circuit. FDNR and AFFDNR specifications and simulation results are summarized in Tables III-IV, Tables V-VI, respectively, and they are shown in Fig. $7-10$.

\section{CONCLUSION}

A new method of noise-shaping gain-filtering is examined with its main components FDNR and AFFDNR. The structure of the proposed filter is basically has two stages. The first part is worked as a third order elliptic FDNR filter providing only one noisy element in the signal path. The designed results are DC gain $80 \mathrm{~dB}$, cutoff frequency $818 \mathrm{KHz}$ and input referred noise power spectral density $5.2 \mathrm{nV} / \mathrm{sqrt}(\mathrm{Hz})$. The second part is gain-filtering stage provided by AFFDNR. The AFFDNR has DC gain $20 \mathrm{~dB}$, cut off frequency $765 \mathrm{KHz}$ and input referred noise power spectral density $83 \mathrm{nV} / \mathrm{sqrt}(\mathrm{Hz})$. Both filters depend on CMOS op amp which has DC gain $82 \mathrm{~dB}$, unity gain bandwidth $319 \mathrm{MHz}$, phase margin 610 and power dissipation $428 \mu \mathrm{W}$.

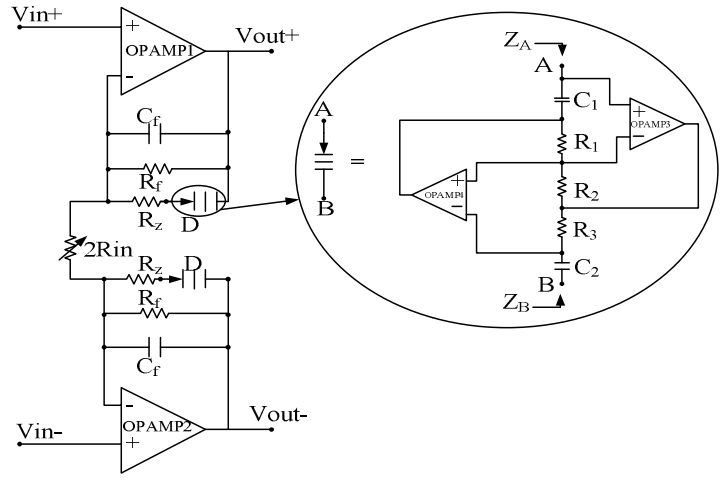

Fig. 6. Instrumentation amplifier with AFFDNR based feedback cascade filter. 
TABLE III. FDNR BASED FILTER SPECIFICATIONS

\begin{tabular}{|c|c|}
\hline Parameters & Expected values \\
\hline DC-gain & $>60 \mathrm{~dB}$ \\
\hline Notch frequency & $\approx 8 \mathrm{MHz}$ \\
\hline Cutoff frequency & $\approx 750 \mathrm{KHz}$ \\
\hline $\begin{array}{c}\text { Input referred noise } \\
\text { power spectral density }\end{array}$ & $\approx 10 \mathrm{nV} / \mathrm{sqrt}(\mathrm{Hz})$ \\
\hline
\end{tabular}

TABLE IV. FDNR BASED FILTER AFTER SIMULATION

\begin{tabular}{|c|c|}
\hline Parameters & Simulation values \\
\hline DC-gain & $80.072 \mathrm{~dB}$ \\
\hline Notch frequency & $7.9667 \mathrm{MHz}$ \\
\hline Cutoff frequency & $792.620 \mathrm{KHz}$ \\
\hline $\begin{array}{c}\text { Input referred noise } \\
\text { power spectral density }\end{array}$ & $5.2335 \mathrm{nV} / \mathrm{sqrt}(\mathrm{Hz})$ \\
\hline
\end{tabular}

TABLE V. AFFDNR CASCADE FILTER SPECIFICATIONS

\begin{tabular}{|c|c|}
\hline Parameters & Expected values \\
\hline DC-gain & $0-20 \mathrm{~dB}$ \\
\hline Cutoff frequency & $\approx 750 \mathrm{KHz}$ \\
\hline $\begin{array}{c}\text { Input referred noise } \\
\text { power spectral density }\end{array}$ & $\approx 14 \mathrm{nV} / \mathrm{sqrt}(\mathrm{Hz})$ \\
\hline
\end{tabular}

TABLE VI. AFFDNR CASCADE FILTER RESULTSAFTER SIMULATION

\begin{tabular}{|c|c|}
\hline Parameters & Simulation values \\
\hline DC-gain & $19.994 \mathrm{~dB}$ \\
\hline Cutoff frequency & $764.707 \mathrm{KHz}$ \\
\hline $\begin{array}{c}\text { Input referred noise } \\
\text { power spectral density }\end{array}$ & $82.624 \mathrm{nV} / \mathrm{sqrt}(\mathrm{Hz})$ \\
\hline
\end{tabular}

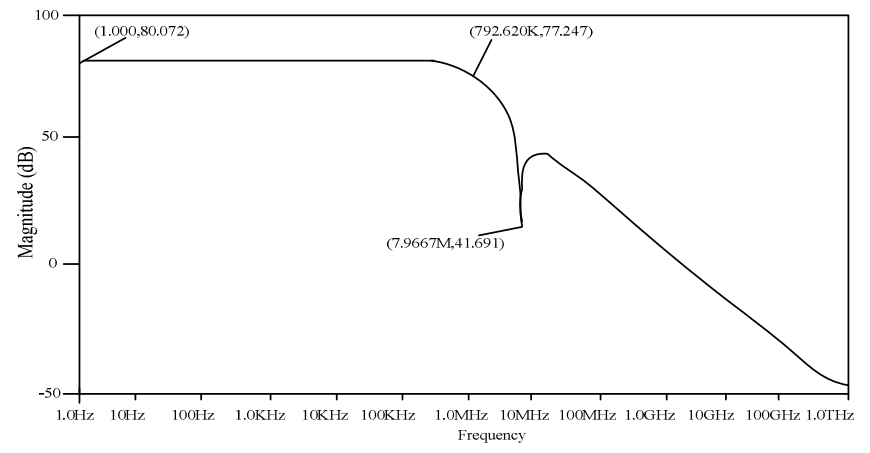

Fig. 7. The magnitude frequency response of FDNR Based Filter.

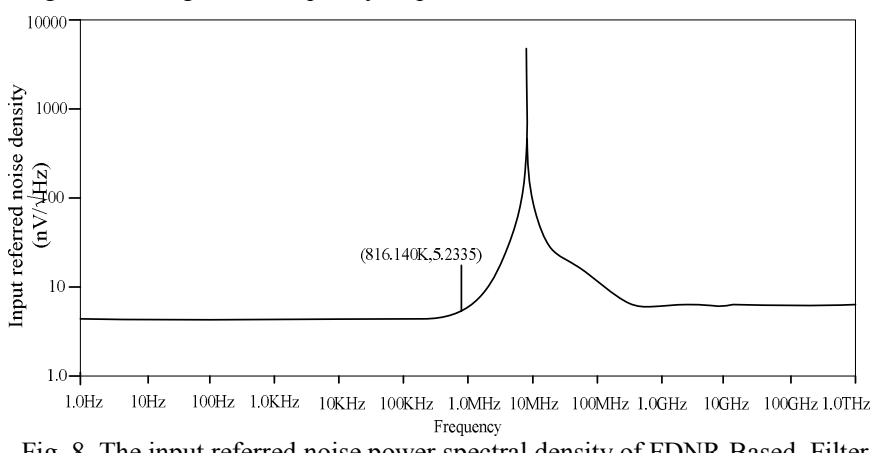

Fig. 8. The input referred noise power spectral density of FDNR Based Filter.

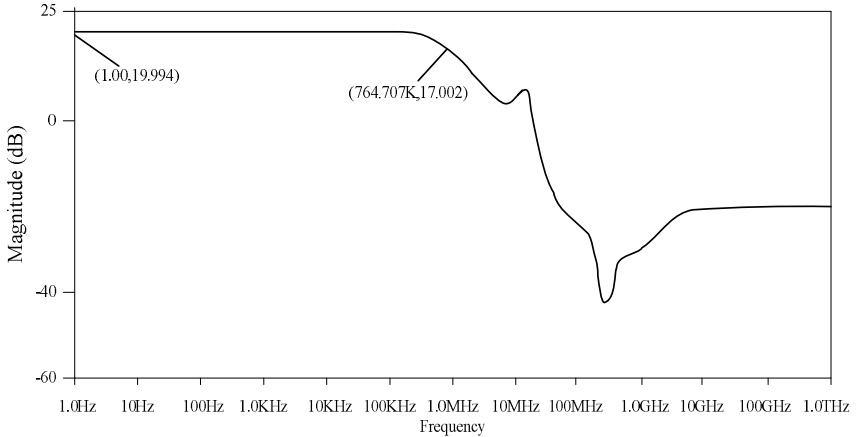

Fig. 9. The magnitude response of AFFDNR Cascade Filter.

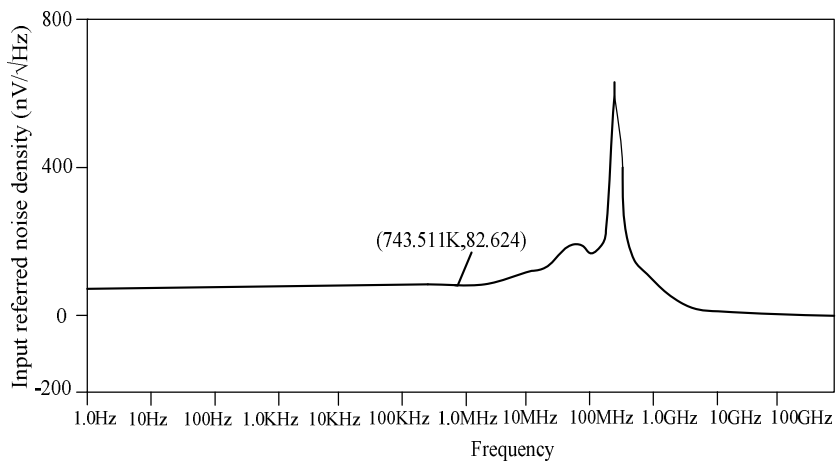

Fig. 10. The input referred noise power spectral density of AFFDNR Cascade Filter.

\section{REFERENCES}

[1] A. Tekin, H. Elwan, A. Ismail and K Pedrotti, "Noise-shaping gainfiltering techniques for integrated receivers", IEEE Journal of SolidState Circuits, vol. 44, no. 10, pp. 2689-2701, October, 2009.

[2] A. Tekin, H. Elwan and K. Pedrotti, "A universal low-noise analog receiver baseband in 65-nm CMOS", Analog IntegrCirc Sig Process, vol. 65 , no. 2 , pp. $225-238,2010$

[3] "Chapter 3 Analog Filter Design", http://cybertron.vlsi.uwindsor.ca/88521/pdf/chapter3.pdf, March 20, 2012, pp. 120-165.

[4] R. J. Baker, CMOS Circuit Design, Layout, and Simulation, 3rd, John Wiley \& Sons, Inc., Hoboken, New Jersey, 2010, pp. 624-652.

[5] H. D. Dammak, S. Bensalem, H. Zouari and M. Loulou, "Design of folded cascode OTA in different regions of operation through $\mathrm{g}_{\mathrm{m}} / \mathrm{I}_{\mathrm{D}}$ methodology", International Journal of Electrical and Computer Engineering, pp. 977- 982, 2008.

[6] S. A. Mahmoud, Ahmed Bamakhramah and Saeed A. Al-Tunaiji, "Low noise Low Pass Filter for ECG Portable Detection Systems with Digitally Programmable Range" in Circuits, Systems, and Signal Processing (CSSP9478), published online: 20 Fab. 2013, doi: 10.1007/s00034-013-9564-9.

[7] T.M. Hassan and S.A. Mahmoud, "New CMOS DVCC Realization and Applications to Instrumentation Amplifier and Active -RC filters" in International Journal of Electronics and Communications, AEU, Vol. 64, pp. 47-55, Jan. 2010. 